\title{
Blind Separation of Chaotic Signals with Multiple Eigenvalue Decomposition Sparse Representation
}

\author{
Liu Sheng ${ }^{1}$, Zhou Shuanghong ${ }^{2}$, Li bing ${ }^{3}$ and Zhang Lanyong ${ }^{4}$ \\ ${ }^{1,3,4}$ College of Automation, Haerbin Engineering University, \\ Haerbin Heilong, 150001China \\ ${ }^{2}$ College of Science, Haerbin Engineering University, \\ Haerbin Heilong, 150001 China \\ 37645694@qq.com
}

\begin{abstract}
For the problem of how to high precision restructure source signals, when laser chaotic signal under the interference of a variety of noise intensities, this paper proposes a blind source separation algorithm based on phase space reconstructed chaotic stream signal. This algorithm firstly makes time delay reconstruction on phase space of separation signals, and then regards the separation matrix as parameter to be optimized, through constructing objective function in phase space to convert blind source separation to an optimization problem, using particle swarm optimization algorithm to solve the optimal separation matrix, and then the observation data is multiplied by optimal separation matrix to reconstruct the source signals. The experimental results show that the algorithm not only has the characteristics of rapid convergence and its accuracy is obviously superior to the existing independent component analysis method under various noise intensities.
\end{abstract}

Keywords: Multi-feature; Blind source separation; Chaotic stream; Particle swarm optimization

\section{Introduction}

Compared with the traditional way of communication, laser chaotic communication has many advantages. Its sensitivity to the initial value and long-term unpredictability, laser chaotic signal is very suitable for secure communication application; the chaotic signal generated by using different chaotic attractors or different initial values and parameters of the same attractor can be thought to be unrelated, therefore, it is easy to generate a large number of mutually orthogonal chaotic signals, which makes the laser chaotic signal has extensive application prospect in multi-user communication [1].However, there are also some challenges may be faced in the application of chaotic technology in laser communication and signal processing, and other fields. For system existing interchannel interference, time-varying fading or hybrid multiuser carrier wave, blind source separation is a problem must be solved. Some researchers using the dynamic property of chaotic signal for blind source separation [2-4], this kind of method can only be applied under the condition of the source signal dynamic equation is known. Independent component analysis (ICA) method was also used to separate chaotic signal [5-7]. This method assumes each source single is mutual statistical and independent, and to separate mixed signal by using high order statistical properties. However, laser chaotic stream signal in essence is to determine the single, and possesses the characteristics of very sensitive to initial value and broadband spectrum, therefore it is difficult to conduct effectively blind source separation only by using statistical properties. This paper uses the theory of phase space reconstruction to depict the problem of blind source separation, puts 
forward a new kind of blind source separation method of chaotic signal aimed at the above problems.

\section{Blind Source Separation Method of Chaotic Signal}

Blind source separation model of laser chaotic stream signal is shown in figure $1, n$ unrelated laser chaotic stream signal $s_{j}(t), j=1, \cdots, n$ are observed by sensor after linear mixture. The signal $x_{i}(t)$ observed by the $i$ th sensor is the linear mixture of each source single $x_{i}(t)=\sum_{j=1}^{n} a_{i j} s_{j}(t)$, and can be expressed in matrix form as :

$$
\boldsymbol{x}(t)=\boldsymbol{A s}(t)+\boldsymbol{n}(t),
$$

Where, $\boldsymbol{x}(t)=\left[x_{1}(t), \cdots, x_{m}(t)\right]^{\mathrm{T}}$ is the observation vector, $\boldsymbol{s}(t)=\left[s_{1}(t), \cdots, s_{n}(t)\right]^{\mathrm{T}}$ is the source vector, the non-singular matrix $\boldsymbol{A}$ constituted by $a_{i j}$ is called hybrid matrix, $\boldsymbol{n}(t)=\left[n_{1}(t), \cdots, n_{m}(t)\right]^{\mathrm{T}}$ is observation noise, here it is assumed as gaussian white noise.

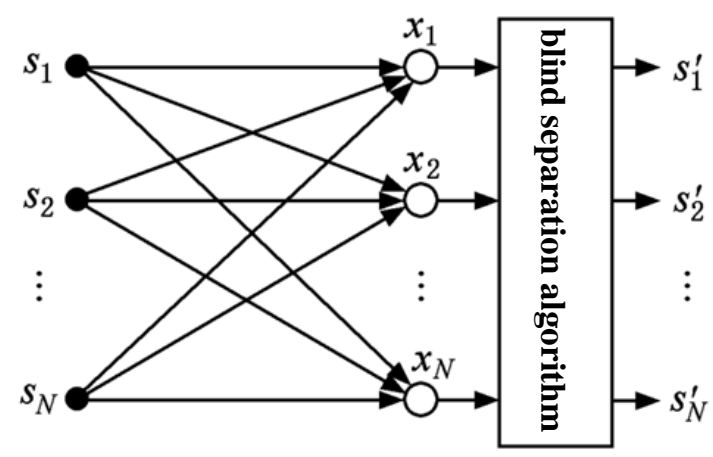

Figure 1. Blind Source Separation Model of Chaotic Stream Signal

According to the observed mixed signal $\boldsymbol{x}(t)$, blind source separation algorithm is trying to get an estimate of the source signal:

$$
\hat{\boldsymbol{s}}(t)=\boldsymbol{B} \boldsymbol{x}(t)=\boldsymbol{B} \boldsymbol{A} \boldsymbol{s}(t)
$$

The matrix $\boldsymbol{B}$ is called as separation matrix. Obviously, if we do not consider the observation noise and $\boldsymbol{B A}=\boldsymbol{I}, \boldsymbol{I}$ is the unit matrix, then the source signal will be perfectly reconstructed. However, due to blind source separation has the problem of inherent fuzziness[8], if the separation matrix satisfying the following equation can be obtained, then it can consider that the mixed signal is successfully separated:

$$
\boldsymbol{B A}=\boldsymbol{\Lambda P},
$$

Where, $\boldsymbol{\Lambda}$ is random $n$-order nonsingular diagonal matrix, $\boldsymbol{P}$ is random $n$-order permutation matrix.

\section{Theory Analysis of Blind Source Separation based on Phase Space Reconstruction}

Phase space reconstruction is widely used in analysis of laser chaotic sequence, such as prediction, diagnosis, and the calculation of Lyapunov index of laser chaotic signal, etc. Literature [9], by using phase space reconstruction, defined index to measure the stream pattern growth rate of phase space of chaotic sequence - growth index. This section will use the growth index to depict blind source separation problem of chaotic signal. 
Assumed that a chaotic sequence $x(t)$ generated by dimension $d_{A}$ chaotic attractor, to estimate the state vector of its phase space by using phase space reconstruction with delay of 1:

$$
\varsigma(t)=[x(t), x(t+1), \cdots, x(t+d-1)]^{\mathrm{T}}, t=1, \cdots, \sigma+1,
$$

Where, $d$ represents delay reconstructed embedded dimension, $\sigma+1$ is the number of state vector obtained from observation sequence. When $d \geq 2 d_{A}$ and are integers, $\varsigma(t)$ can smoothly reconstruct the chaotic attractor. In blind source separation problem, dimension $d_{A}$ of each source chaotic stream signal generally cannot be known in advance, for better restore the properties of chaotic attractor, $d$ should be taken as large as possible, when the observation sequence length is $N$, for example, you can take $d=N-\sigma$.In phase space, the distance between $\varsigma(t+1)$ and $\varsigma(1)$ is defined as follows:

$$
V_{\varsigma}(t)=\|\varsigma(t+1)-\varsigma(1)\|^{2},
$$

Where, $\|\cdot\|$ is the norm of $l^{2}$. Different laser chaotic signal has different growth rate in steam pattern of phase space, it can be described by defining the growth index:

$$
P_{\sigma}(\varsigma)=\frac{D\left(V_{\varsigma}(t)\right)}{E^{2}\left(V_{\varsigma}(t)\right)}, t=1, \cdots, \sigma,
$$

Where, $D(\square)$ represents variance, $E(\square)$ represents mathematical expectation, $\sigma$ is a control parameter, and when there is no noise, $\sigma=5$ to decrease the computation burden of $P_{\sigma}(\varsigma)$, observation noise is $\sigma=100$ when it should not be neglected.

Literature [9] proved that, when observation sequence of reconstructed $\varsigma(t)$ has zeromean, $P_{\sigma}(\varsigma)$ has the following properties:

Property 1 scale invariance, that is $P_{\sigma}(\varsigma)=P_{\sigma}(k \varsigma), \forall k \neq 0$.

Property $2 P_{\sigma}\left(k_{1} \varsigma_{1}+k_{2} \varsigma_{2}\right)<\max \left(P_{\sigma}\left(\varsigma_{1}\right), P_{\sigma}\left(\varsigma_{2}\right)\right), \forall k_{1}, k_{2} \neq 0$, where $\varsigma_{1}$ and $\varsigma_{2}$ are two irrelevant random sequences.

$k_{1} \varsigma_{1}(t)+k_{2} \varsigma_{2}(t)$ 。Assumed that we have irrelevant observation sequence $x_{1}(t)$ and $x_{2}(t)$, to carry on phase space reconstruction with time delay of 1 on it to obtain state vector $\varsigma_{1}(t)$ 和 $\varsigma_{2}(t), t=1, \cdots, \sigma+1$, respectively, therefore, the state vector of sequence $k_{1} x_{1}(t)+k_{2} x_{2}(t) a$ reconstruction is $k_{1} \varsigma_{1}(t)+k_{2} \varsigma_{2}(t)$ Combined with the property 2 of $P_{\sigma}(\varsigma)$ two can get the following conclusion: irrelevant time sequence produced by two different dynamic systems, the growth index of its linear combination is always less than one of the two sequence time with a larger growth index; any one sequence of $n$ irrelevant sequences is irrelevant to the linear combination of the rest $n-1$ sequences, so the growth index of a linear combination of $n$ irrelevant sequences is always less than one of these sequences with a largest growth index. This can inspire us that, we can convert the blind source separation problem containing $n$ mixed signals into an optimization problem with orthogonal constraint:

$$
\max _{\boldsymbol{B} \in R^{n \times n}}\left\{P_{\sigma}\left(\boldsymbol{\varsigma}_{\hat{s}, 1}\right)+, \cdots,+P_{\sigma}\left(\varsigma_{\hat{s}, n-1}\right)\right\} \quad \text { subject to } E\left(\hat{\boldsymbol{s}}(t) \hat{\boldsymbol{s}}^{\mathrm{T}}(t)\right)=\boldsymbol{I},
$$

Where, $\boldsymbol{B}$ is separation matrix, $P_{\sigma}\left(\varsigma_{\hat{s}, i}\right)$ is the growth index of reconstructed $i$ channel source single $\hat{s}_{i}(t)$, constraint condition $E\left(\hat{\boldsymbol{s}}(t) \hat{\boldsymbol{s}}^{\mathrm{T}}(t)\right)=\boldsymbol{I}$ ensures the obtained each channel separation signal $\hat{s}_{i}(t)$ is orthogonal with solution space of other solved separation signal $\hat{s}_{j}(t), j \neq i$. 


\section{Blind Source Separation Algorithm Process}

The property 1 of growth index in previous section only set up on zero mean, therefore, it shall do mean value removal of observation signal first:

$$
x_{i}(t)=x_{i}(t)-E\left(x_{i}(t)\right), i=1, \cdots, n,
$$

Property 2 of growth index further requires the signal sequence is irrelevant with each other, which can be realized by pre-whitening observation signal. Assumed that autocorrelation matrix of $\boldsymbol{x}(t)$ characteristic decomposition $E\left(\boldsymbol{x}(t) \boldsymbol{x}^{\mathrm{T}}(t)\right)=\boldsymbol{Q} \boldsymbol{\Sigma} \boldsymbol{Q}^{\mathrm{T}}$, where, $\boldsymbol{Q}$ is orthogonal matrix, $\boldsymbol{\Sigma}$ is diagonal matrix, then $\boldsymbol{W}=\Sigma^{-1 / 2} \boldsymbol{Q}^{\mathrm{T}}$ is called as whitening matrix, after linear conversion $\not \partial t(t)=\boldsymbol{W} \boldsymbol{x}(t)$, each component of $\tilde{\boldsymbol{x}}(t)$ is irrelevant with each other.

By Cayley conversion, any $n$-order orthogonal matrix can be decomposed into a series of the product of the rotation matrixes, and contains parameter vector $\boldsymbol{\theta}=\left\{\theta_{1}, \cdots, \theta_{n(n-1) / 2}\right\}$ of $n(n-1) / 2$ parameters to express [20]. This method can reduce the parameter to be optimized from $n^{2}$ to $\left(n^{2}+n\right) / 2$, the convergence speed and robustness of the algorithm is improved significantly. Subsequent sections of this part adopt the following forms of parameterization to express 2 and 3-order orthogonal matrix:

$$
\begin{gathered}
\boldsymbol{B}_{2 \times 2}=\left[\begin{array}{cc}
\cos \theta_{1} & -\sin \theta_{1} \\
\sin \theta_{1} & \cos \theta_{1}
\end{array}\right], \\
\boldsymbol{B}_{3 \times 3}=\left[\begin{array}{ccc}
1 & 0 & 0 \\
0 & \cos \theta_{1} & -\sin \theta_{1} \\
0 & \sin \theta_{1} & \cos \theta_{1}
\end{array}\right]\left[\begin{array}{ccc}
\cos \theta_{2} & 0 & -\sin \theta_{2} \\
0 & 1 & 0 \\
\sin \theta_{2} & 0 & \cos \theta_{2}
\end{array}\right]\left[\begin{array}{ccc}
\cos \theta_{3} & -\sin \theta_{3} & 0 \\
\sin \theta_{3} & \cos \theta_{3} & 0 \\
0 & 0 & 1
\end{array}\right],
\end{gathered}
$$

Where, parameter vector $\theta_{i} \in[0,2 \pi]$. Using this parameter expression, can convert (7) into an unconstrained optimization problem :

$$
\max _{\theta_{i} \in[0,2 \pi]}\left\{P_{\sigma}\left(\varsigma_{y, 1}\right)+, \cdots,+P_{\sigma}\left(\varsigma_{y, n-1}\right)\right\}, i=1, \cdots, n(n-1) / 2,
$$

For an unconstrained optimization problem, particle swarm optimization algorithm [10] is a very effective method. The parameter vector $\boldsymbol{\theta}=\left\{\theta_{1}, \cdots, \theta_{n(n-1) / 2}\right\}$ is regarded as the particle position, the objective function (11) is regarded as fitness function, can use particle swarm optimization algorithm to estimate the optimal separation matrix $\boldsymbol{B}_{\text {opt }}$, so as to reconstruct each channel source signal. The whole algorithm process is shown in figure 2:

1. Mean-value removal of observation signal $\boldsymbol{x}(t)$;

2. Pre-whitening of observation signal $\boldsymbol{x}(t)$ to obtain $\tilde{\boldsymbol{x}}(t)$;

3. Using the random number uniform distributed on $[0,2 \pi]$ to initialize the initial position of each particle;

4. For each particle, according to the (9), (10) to calculate separation matrix B, and calculate separation signals by (2), carry on phase space reconstruction according to (4) and calculate the fitness function value according to (5) to (7);

5. Using the particle swarm algorithm to optimize (11), until the termination condition is met, record the optimal particle position $\boldsymbol{\theta}$ opt ;

6. To calculate separation matrix Bopt according to (9) and (10), and output reconstructed source signal $\boldsymbol{y}(t)=\boldsymbol{B}$ opt $\tilde{\boldsymbol{x}}(t)$.

Figure 2. Blind Source Separation Algorithm Flow Chart 


\section{Simulation Experiment}

This section will evaluate the performance of blind source separation algorithm through Matlab simulation experiment. Source signal generates mixed signal by (1), elements of mixed matrix $\mathrm{A}$ is generated independently and randomly by uniform distribution of $[1,1]$ in each simulation. The accuracy of blind source separation algorithm will be measured through performance index (PI):

$P I=\frac{1}{n(n-1)} \sum_{i=1}^{n}\left(\sum_{j=1}^{n} \frac{\left|g_{i j}\right|^{2}}{\max _{k}\left|g_{i k}\right|^{2}}\right)+\sum_{j=1}^{n}\left(\sum_{i=1}^{n} \frac{\left|g_{i j}\right|^{2}}{\max _{k}\left|g_{k j}\right|^{2}}\right)$,

Where, $\boldsymbol{G}=\boldsymbol{B} \boldsymbol{W A}$, the smaller of $P I$ the better, when $\boldsymbol{G}$ satisfies (3), PI gets the minimal value 0 .

Four source signals used in simulation experiments, are generated respectively by Rossler attractor (13), Lorentz attractor (14), Duffing attractor (15) and Mackey-Glass attractor (16), the first three signals are generated through the integration of the fourth order runge kutta method, integral step length are $0.05,0.05,0.01$, respectively, the length of the signal is $N=2000$, as shown in figure 3. The four signals are divided into two groups, are used to test the blind source separation effects of three-channel mixed signals and two-channel mixed signal, as shown in Table 1.

$$
\begin{gathered}
\left\{\begin{array}{l}
d x / d t=-y-z \\
d y / d t=x+0.2 y \\
d z / d t=0.2+z(x-5)
\end{array}\right. \\
\left\{\begin{array}{l}
d x / d t=16(y-x) \\
d y / d t=45.92 x-y-x z \\
d z / d t=x y-4 z
\end{array}\right. \\
\left\{\begin{array}{l}
x / d t=y \\
d y=-0.5 y+x-x^{3}+0.42 \sin t
\end{array}\right. \\
x(i+1)=x(i)+\frac{0.2 x(i-17)}{1+x^{10}(i-17)}-0.1 x(i)
\end{gathered}
$$

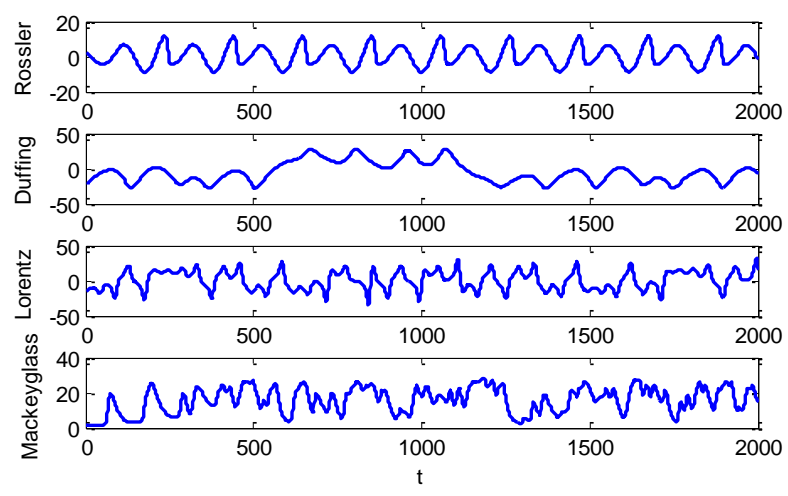

Figure 3. Source Signal Waveform 
Table 1. Test Signal Grouping

\begin{tabular}{cc}
\hline source signal of group 1 & Rossler、Lorentz、Duffing \\
source signal of group 2 & Mackey-Glass、Duffing \\
\hline
\end{tabular}

\subsection{Simulation Results of Blind Source Separation of Chaotic Stream Signal at No Noise}

For the three-channel source signal in group 1, to carry on four blind source separation experiments with the method proposed in this paper, performance index $P I$ changes with the change of iterative steps as shown in figure 4 . With the increase of iteration steps, $P I$ decreases rapidly, and in all experiments, algorithm can be convergence through dozens of iterations. Table 2 shows the experimental results after 100 times of Monte Carlo Simulations on two groups of source signal, it can be seen that, both for the separation of three-channel mixed signals in group 1 or two-channel mixed signal in group 2, the separation accuracy of the proposed algorithm is better than fast independent component analysis (FastICA)[6].

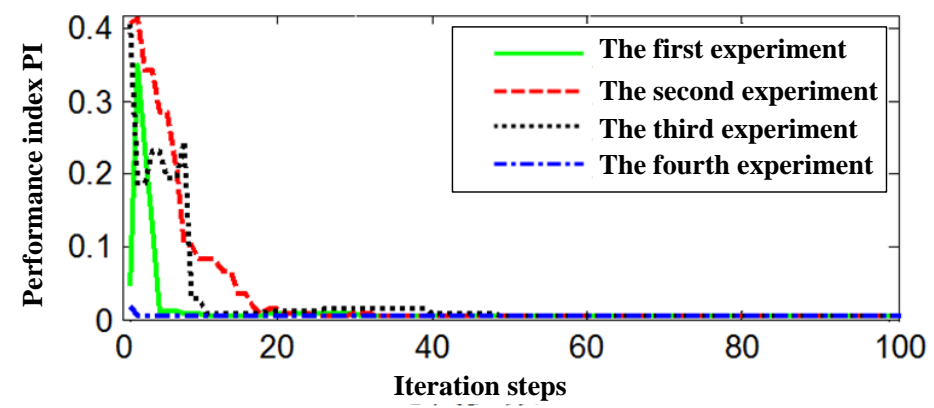

Figure 4. The Convergence Condition of the Algorithm at No Noise

Table 2. The Blind Source Separation Performance at No Noise

\begin{tabular}{ccc}
\hline \multirow{2}{*}{ Separation method } & \multicolumn{2}{c}{ test signal } \\
\cline { 2 - 3 } & Group 1 & Group 2 \\
\hline Proposed algorithm & 0.0082 & 0.0056 \\
FastICA & 0.0728 & 0.0172 \\
\hline
\end{tabular}

\subsection{Blind Source Separation Simulation Results of Laser Chaotic Stream Signal under Noise Environment}

This section will evaluate the blind source separation performance of algorithm to noise contaminated mixed signal, it is assumed that the observation noise is the zero mean gaussian white noise. Let the observation noise changes from $20 \mathrm{~dB}$ to $50 \mathrm{~dB}$, take a test point at the interval of $5 \mathrm{~dB}$, repeat 100 times of Monte Carlo simulation on each test point, and results as shown in figure 5 . 

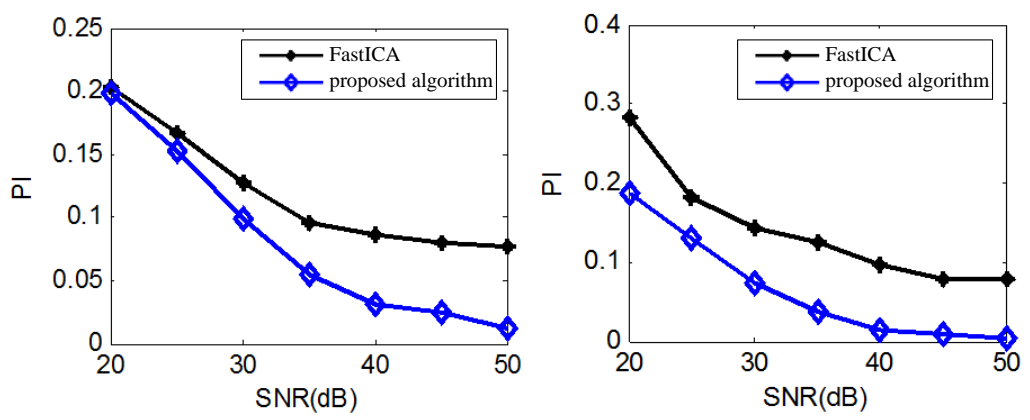

\section{Figure 5. The Performance of Blind Source Separation Algorithm under Different Noise Intensities}

\subsection{Comparison between the Proposed Algorithm and other Dictionary Learning Algorithms}

The experiment in this section mainly includes a comparison between the proposed algorithm in this paper and traditional dictionary learning algorithms $\mathrm{K}$ SVD and RLS-DLA in terms of de-noising effect. 100 particles were selected for PSO used in this paper. 100 iterations were given.

The wave form of the selected signal to be observed is Fig. 6(a); upon provision of noise, the signal-noise ratio is $10 \mathrm{~dB}$, with the wave form shown in Fig. 6(b). Denoising is achieved with three algorithms, with the results as follows:

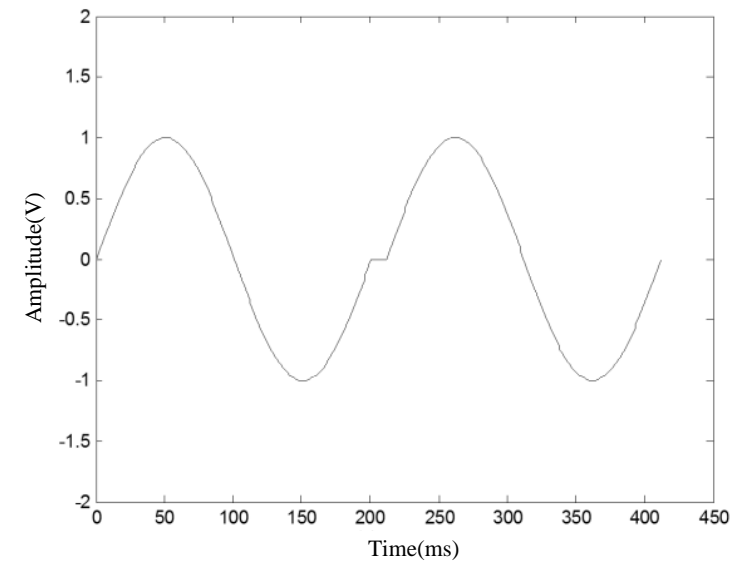

(a) Wave form of signal without noise

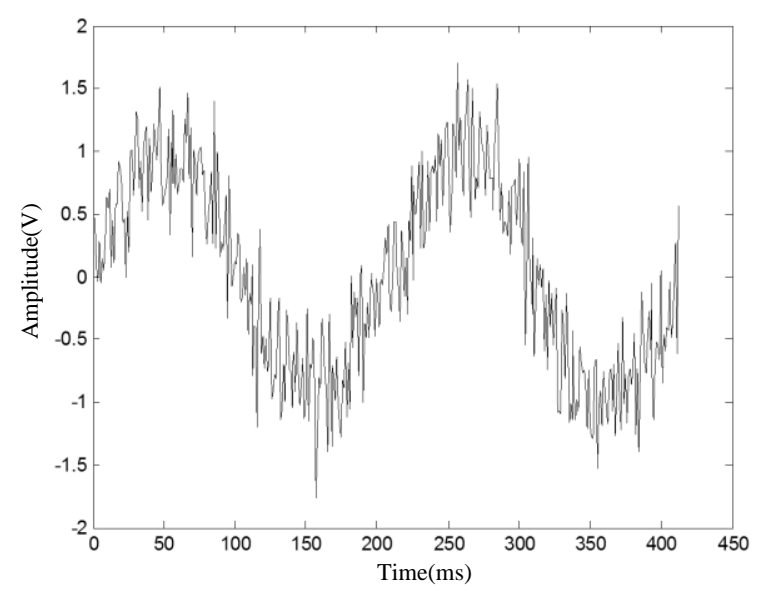

(b) Wave form of signal with noise 


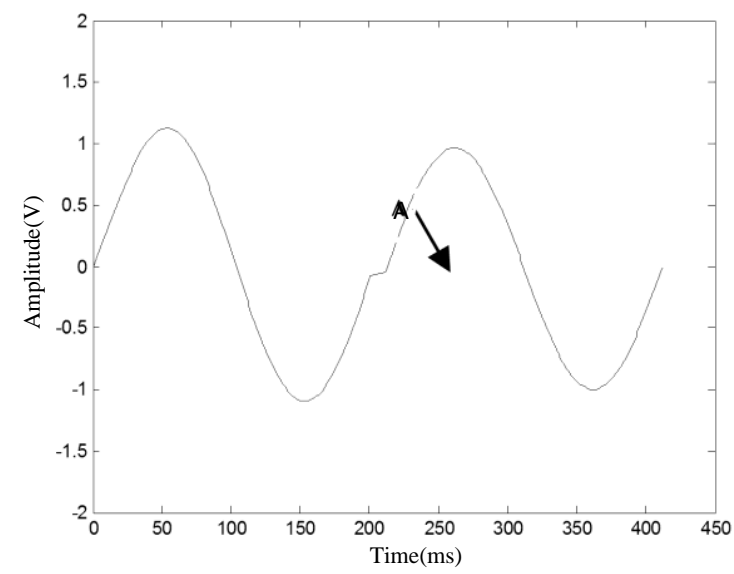

(c) Signal reconstructed by the proposed algorithm

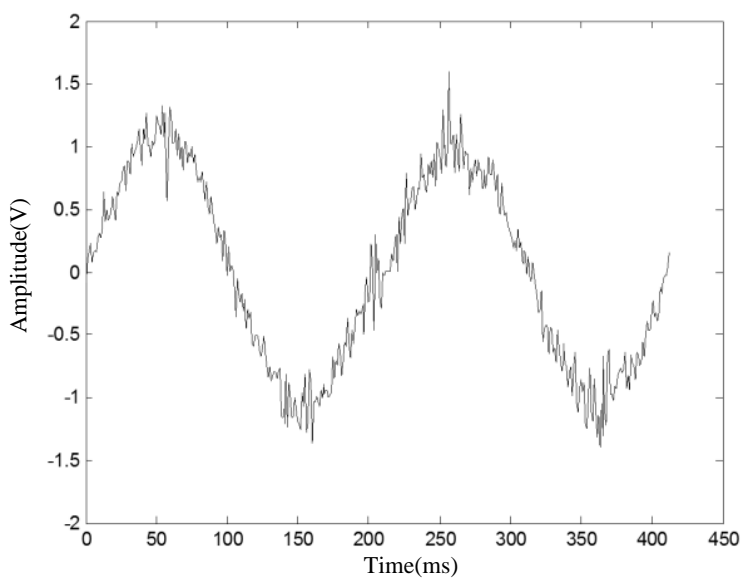

(d) Signal reconstructed by K-SVD algorithm

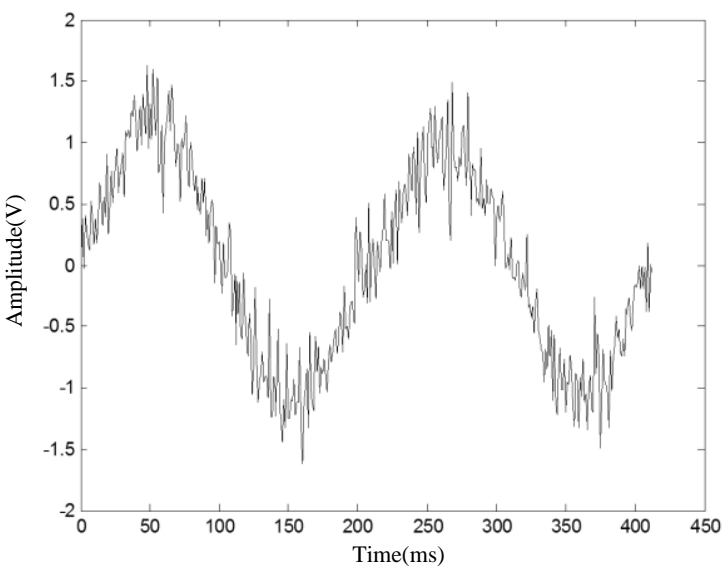

(e) Signal reconstructed by RLS-DLA algorithm

Figure 6. Comparison between Reconstructed Signals

From point A, we learned that the proposed algorithm provided better results in terms of detail processing as PSO was used for sparse solving following which the sparse vector was obtained, which facilitated the recovery of the signal's detail features. 


\subsection{Application of the Proposed Algorithm to Radar Echo Signal}

For the experiment on de-noising effect of electromagnetic signal using the proposed algorithm, the point target echo signal of LMP (linear frequency modulation) radar was selected for de-noising. The results were shown in Fig. 7. Upon calculation of the de-noised signal-noise ratio, the result was $\mathrm{SNR}=25.121 \mathrm{~dB}$, $\mathrm{MSE}=0.0126$. It showed that the proposed algorithm de-noised radar signal in a better way. This is an effective de-noising solution.
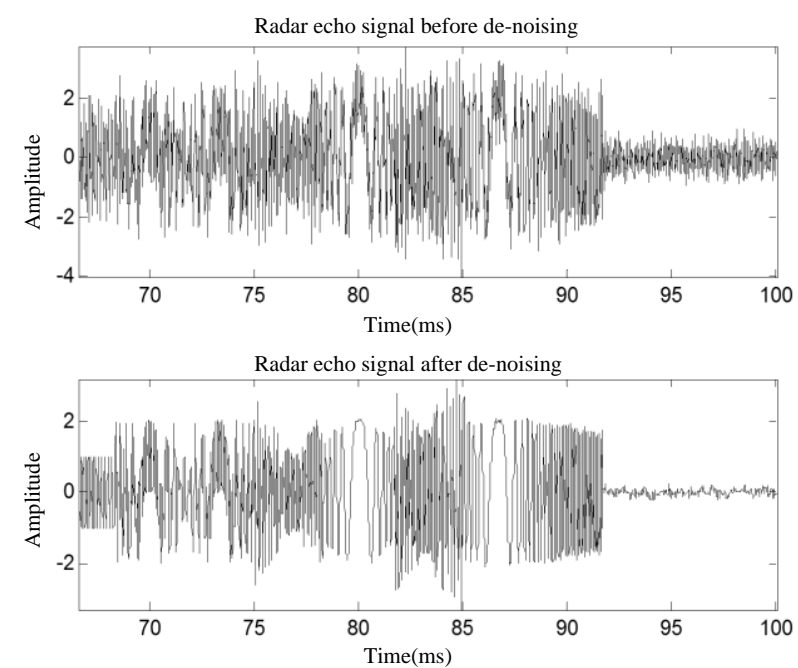

Figure 7. De-noising Experiment of Radar Echo Signal

\section{Conclusion}

This paper depicts the blind source separation using the theory of phase space reconstruction, and proposes a new blind source separation algorithm for laser chaotic stream signal. The algorithm constructs the objective function in the phase space of separation signal, to convert the blind source separation of chaotic stream signal into an unconstrained optimization problem, and be solved through the particle swarm optimization algorithm. At the same time, it adopts parametric representation of orthogonal matrix on separation matrix, effectively reduces the dimension of the optimization problem, therefore, the algorithm can be quickly converged. Simulation results show that the algorithm not only has the characteristics of rapid convergence, and its accuracy under various SNR is obviously better than that of FastICA algorithm.

\section{References}

[1] Gan Yan, Yuxiang Lv, Qiyin Wang, Yishuang Geng, Routing algorithm based on delay rate in wireless cognitive radio network, Journal of Networks, 9(4), 948-955, Jan. (2014).

[2] Lin Y, Yang J, Lv Z. A Self-Assessment Stereo Capture Model Applicable to the Internet of Things[J]. Sensors, (2015), 15(8): 20925-20944.

[3] Wang K, Zhou X, Li T. Optimizing load balancing and data-locality with data-aware scheduling[C]. Big Data (Big Data), 2014 IEEE International Conference on. IEEE, (2014): 119-128.

[4] Zhang L, He B, Sun J. Double Image Multi-Encryption Algorithm Based on Fractional Chaotic Time Series[J]. Journal of Computational and Theoretical Nanoscience, (2015), 12: 1-7.

[5] Su T, Lv Z, Gao S. 3d seabed: 3d modeling and visualization platform for the seabed[C]. Multimedia and Expo Workshops (ICMEW), 2014 IEEE International Conference on. IEEE, (2014): 1-6. 
[6] Yishuang Geng, Jin Chen, Ruijun Fu, Guanqun Bao, Kaveh Pahlavan, Enlighten wearable physiological monitoring systems: On-body rf characteristics based human motion classification using a support vector machine, IEEE transactions on mobile computing, 1(1), 1-15, Apr. (2015).

[7] Lv Z, Halawani A, Feng S. Multimodal hand and foot gesture interaction for handheld devices[J]. ACM Transactions on Multimedia Computing, Communications, and Applications (TOMM), (2014), 11(1s): 10.

[8] Guanxiong Liu, Yishuang Geng, Kaveh Pahlavan, Effects of calibration RFID tags on performance of inertial navigation in indoor environment, 2015 International Conference on Computing, Networking and Communications (ICNC), Feb. (2015).

[9] Jie He, Yishuang Geng, Yadong Wan, Shen Li, Kaveh Pahlavan, A cyber physical test-bed for virtualization of RF access environment for body sensor network, IEEE Sensor Journal, 13(10), 3826-3836, Oct. (2013).

[10] Wenhua Huang, Yishuang Geng, Identification Method of Attack Path Based on Immune Intrusion Detection, Journal of Networks, 9(4), 964-971, Jan. (2014).

[11] Li X, Lv Z, Hu J. XEarth: A 3D GIS Platform for managing massive city information[C]. Computational Intelligence and Virtual Environments for Measurement Systems and Applications (CIVEMSA), 2015 IEEE International Conference on. IEEE, (2015): 1-6.

[12] Lv, Z., Réhman, S. U., \& Chen, G. (2013, November). Webvrgis: A p2p network engine for vr data and gis analysis. In International Conference on Neural Information Processing (pp. 503-510). Springer Berlin Heidelberg.

[13] Lu, Z., Réhman, S. U., \& Chen, G. (2013, September). Webvrgis: Webgis based interactive online 3d virtual community. In Virtual Reality and Visualization (ICVRV), 2013 International Conference on (pp. 94-99). IEEE.

[14] Zhang, M., Lv, Z., Zhang, X., Chen, G., \& Zhang, K. (2009). Research and Application of the 3D Virtual Community Based on WEBVR and RIA. Computer and Information Science, 2(1), 84.

[15] Lv, Z., Feng, L., Li, H., \& Feng, S. (2014, November). Hand-free motion interaction on google glass. In SIGGRAPH Asia 2014 Mobile Graphics and Interactive Applications (p. 21). ACM.

[16] Lv, Z., Feng, S., Feng, L., \& Li, H. (2015, March). Extending touch-less interaction on vision based wearable device. In 2015 IEEE Virtual Reality (VR) (pp. 231-232). IEEE.

[17] Ou, W., Xie, Z., \& Lv, Z. (2015, October). Spatially Regularized Latent topic Model for Simultaneous object discovery and segmentation. In Systems, Man, and Cybernetics (SMC), 2015 IEEE International Conference on (pp. 2938-2943). IEEE.

\section{Authors}

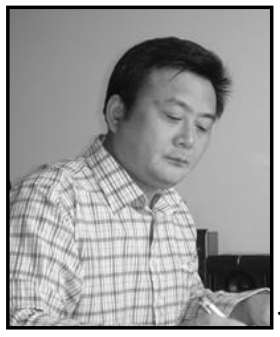

Liu Sheng, $\mathrm{He}$ is engineering, Ph.D and professor of Harbin engineering university institute of automation. His research interest is mainly in the area of Stochastic system optimal estimation and control,Ship navigation and attitude control, Robust control system theory and application, Design and implementation of automatic control system. He has published many research papers in scholarly journals in the above research areas and has published several books.

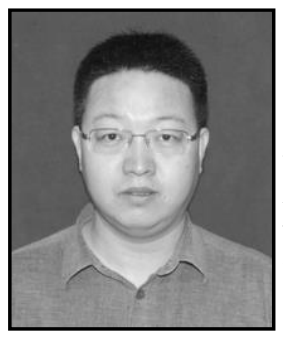

Zhou Shuanghong, He received his M.S. degree in science college of Harbin engineering university in Harbin, China. He is currently a lecturer in the College of science at Harbin engineering university. His research interest is mainly in the area of Signal processing, Electromagnetic compatibility and Numerical calculation. He has published several research papers in scholarly journals in the above research areas and has participated in several books. 